\title{
Youth sport and COVID-19: a potential generation lost
}

\section{[version 1; peer review: 2 not approved]}

\section{Murray Drummond (D), Sam Elliott(D), Claire Drummond, Ivanka Prichard}

SHAPE Research Centre, Flinders University, Bedford Park, SA, 5000, Australia

V1 First published: 19 May 2020, 2:27

https://doi.org/10.35241/emeraldopenres.13661.1

Latest published: 19 May 2020, 2:27

https://doi.org/10.35241/emeraldopenres.13661.1

Open Peer Review
Approval Status
United Kingdom
2. Bente Wold, University of Bergen, Bergen,
Norway
Any reports and responses or comments on the
article can be found at the end of the article.

\section{Abstract}

This conceptual / study protocol paper provides important context around the role of sport in Australia where sport provides aspects of community agency through participation, organisation and volunteerism. It provides a descriptive analysis of how sport assists young people in developing physical and mental "fitness" through its community orientation. However, it also provides discussion around the potential of a "generation lost" to sport as a consequence of the coronavirus disease 2019 (COVID-19) pandemic. The conceptual nature of this paper means that the data collection underpinning this research has not yet been conducted. However, given that we have applied for human research ethics along with having accrued sporting clubs and organisations eager to be involved in the research, we are planning to roll out this research by mid 2020 . The design will be based on mixed methods approach whereby large-scale surveys together with focus groups and interviews will be central to the research data collection process. This research is unique given the nature of the time in which it exists. The last global pandemic was around 100 years ago when sport did not play such a significant role in society. Understanding the implications of the pandemic on young people and to the sporting clubs and organisations will be key in reestablishing sport as a central component of community agency towards the physical and mental health of young people. The urgency of understanding this is key to assisting the loss of potential young people to sport and the benefits that go with it to the individual, the community, and society as a whole.

\section{Keywords}

COVID-19, Pandemic, Youth sport, community sport, mixed methods research, sport dropout.

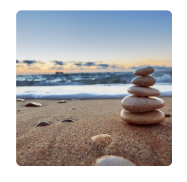

This article is included in the Healthier Lives

gateway. 
Author roles: Drummond M: Conceptualization, Methodology, Writing - Original Draft Preparation, Writing - Review \& Editing; Elliott S: Conceptualization, Methodology, Writing - Original Draft Preparation, Writing - Review \& Editing; Drummond C: Conceptualization, Methodology, Writing - Original Draft Preparation, Writing - Review \& Editing; Prichard I: Investigation, Methodology, Writing - Original Draft Preparation, Writing - Review \& Editing

Competing interests: No competing interests were disclosed.

Grant information: The author(s) declared that no grants were involved in supporting this work.

Copyright: @ 2020 Drummond $\mathrm{M}$ et al. This is an open access article distributed under the terms of the Creative Commons Attribution License, which permits unrestricted use, distribution, and reproduction in any medium, provided the original work is properly cited.

How to cite this article: Drummond M, Elliott S, Drummond C and Prichard I. Youth sport and COVID-19: a potential generation lost [version 1; peer review: 2 not approved] Emerald Open Research 2020, 2:27 https://doi.org/10.35241/emeraldopenres.13661.1

First published: 19 May 2020, 2:27 https://doi.org/10.35241/emeraldopenres.13661.1 


\section{Introduction}

The global impact of the COVID-19 Pandemic will forever impact sport in the lives of children and young people. Like other nations, Australia is amidst an unprecedented challenge that threatens to uproot the traditions, systems and opportunities that have long been stable artefacts of community sport. In line with "social distancing" principles stipulated by the Australian Government, sporting clubs and organizations have had to cease all operations and this has the potential to create a significant risk to longevity in the event of a long-term hiatus. It also means that a generation of young people may be lost to sport because of the global disruption to sport.

Australia is a nation that, like many others, prides itself on its sporting prowess. For a relatively small population of around 25.5 million (ABS, 2020) it has historically been, and continues to be, an important part of the international sporting "landscape". In using a sporting metaphor, as many Australians say, "we punch above our weight" where sport is concerned. However, sport in Australia, other than some elite professional sports, is largely run and organised at the community level, and is highly dependent upon volunteers along with financial donations and minimal government subsidies (Cuskelly, 2004). Clearly some sports perform better than others in terms of participation rates and as a consequence they are more financial, and seemingly well, positioned. Cricket, netball, Australian football, and both Ruby League and Rugby Union are sports that thrive in Australia and maintain high levels of participants from juniors through to seniors (ABS, 2012). They are also the sports that receive the majority of media exposure through television coverage. However, men's sports, like most nations around the world, receive the most coverage along with funding and general exposure (Caple et al., 2011). Regardless, these are also the sports with strong junior pathways and, for those that are internationally played such as cricket, netball and Rugby Union, the pathways have held the nation in good stead with many world-dominating performances; such as Ashes Cricket success, Rugby World Cup victories and netball world championships. The problem is that many sporting clubs and organizations are not well prepared for a pandemic or sporting hiatus of the magnitude that COVID-19 has created. Consequently, there could be many young people who will drop out of their current sport, and potentially be lost to sport forever, if we do not plan for a structured way forward both now and following the pandemic.

\section{A potential generation lost}

Given that most volunteers in youth sport are parents, the necessity to return to work once the pandemic recedes may result in the availability of fewer volunteers to support organized sport programs. Being a team coach, manager or secretary demands a significant time commitment, which might be improbable for many working families; especially those in low socioeconomic positions, and those seeking to transition back into the workforce after a period of unemployment. Families may also be enduring a mode of 'cost recovery' if they choose to 'freeze' mortgage repayments during the pandemic or were forced into other modes of financial survival (e.g. drawing on superannuation). Consequently, many families, especially those in remote communities and geographic regions with low population density, may reduce their voluntary efforts altogether, which places an enormous strain on the reemergence of organized youth sport. Even if clubs and organizations can 'keep the doors open', a strategy to re-engage parent volunteers appears a critical factor if youth sports are to involve trainings and competitions into the future.

A compounding issue is that many sporting clubs will be financially stricken which might force clubs to maintain or even increase seasonal fees and registrations. Even before the pandemic, cost was readily identified as a barrier to sport participation for low-income families (Holt Kingsley et al., 2011). From a club and organizational perspective, the need to pay lease agreements and redundancies will only emphasis the need for seasonal registration fees in order for clubs and organizations to remain viable. That is of course, without government assistance. Consequently, a hiatus from youth sport is likely to endure beyond the 'social distancing' period for low-income, unemployed and underemployed families who are unable to meet the financial demands that enable youth sport participation. It might also mean that many high cost sporting activities experience a sharp decline in participation with children from lowand middle-income families. This should prompt sporting clubs and organizations to consider how they might plan to re-engage families which, if ignored, could result in low-cost sporting options gaining popularity at the literal expense of other high cost options, or at worst, losing families and youth participants altogether. Revitalizing the youth sport sector may therefore have unanticipated consequences for families who were already 'at risk' of dropping out from sport.

Talent pathways and elite development programs for youth athletes are also likely to be impacted by seismic changes to professional sport. The pathway to national competitions such as the Australian Football League (AFL), Netball Australia's Super Netball and National Rugby League (NRL), for instance, has typically fostered large numbers of emerging youth athletes in targeted 'feeder' pathways to the professional level (Haycraft et al., 2017). However, the COVID-19 Pandemic has forced indefinite 'hibernation' of sporting organizations and clubs across the nation, and globally, which has resulted in significant job losses within the industry. It is forecast that in many of the most professionally organised sports such as AFL, which is a billion dollar industry, clubs will be forced to reduce operational expenditure and consequently the size of playing lists (AFL, 2020). This is a problem for youth participants in elite pathways because the aspiration to make the elite level - a key motivational driver for their sustained involved in specialized sport programs - is now made more difficult given that fewer opportunities exist. Subsequently, several talented youth athletes may drop out from talent pathway programs altogether if they feel the window of opportunity is near impossible. Anecdotally, this appears to be a key concern for many youth athletes situated in their 'draft year' - the year when they would normally enter the 'draft' in hope of being recruited to a national team. 
In recent times, sport in Australia has shifted focus toward a more inclusive agenda to maximize participation but these efforts may be thwarted as a result of the Pandemic. The establishment of innovative, youth sport programs and theoretical models designed to improve participation and retention among girls (Elliott et al., 2019), ethnically diverse groups (Jeanes et al., 2019), First Nations Australians (Ferrer \& Turner, 2017), and children with a disability (Jeanes et al., 2019) has become a key strategic priority for community, state-level and national teams and organizations. This coincided with newly created positions of employment such as a 'Disability and Inclusion Officer' or 'Female Pathways Development Officer' to oversee game development and expansion. However, the Pandemic has resulted in most administrative and service roles being 'stood down' or 'disestablished', placing the longevity of these programs at risk. Given the importance of widening participation as well as the need to restart youth sport programs in a viable manner, it is plausible that all these individual programs may be effectively 'bundled' together in a single role. The incumbent employed to oversee these programs will be required to manage a broader framework of inclusion. Without developing new efficiencies to support this agenda, it is likely that many of the inclusion programs that are vital to engaging minority groups of youth sport participants will not have the same reach and impact as a result of fewer resources available. The inevitable outcome will be a failure to maximize participation, further contributing to the idea of a potential generation lost to sport.

Prior to the COVID-19 pandemic, a number of sporting clubs and organizations were exploring new ways to facilitate mental health literacy messages to their sporting community (for example, Dowell et al., 2020). It was not uncommon for teams to participate in important educational sessions about depression, drugs and alcohol and domestic violence as part of a holistic approach to developing youth athletes. Likewise, many wellresourced clubs and organizations spent considerable resources engaging with schools and communities to deliver important health-related messages about nutrition, sleep and physical activity (see Drummond et al., 2016). However, these programs may cease to exist if (a) the clubs cease to exist, (b) the volunteers who champion these initiatives are lost to sport, (c) clubs no longer have the resources to invest in these ancillary programs or (d) club priorities and missions change in response to the emergence of a 'new' participatory market. This is a potentially significant issue for families seeking to enroll their child in sport environments that provide holistic developmental support. It may not be a primary determinant but could be a decisive factor that influences parents and children's decision-making about which sport and club/team they wish to join, especially if their former club/team ceases to exist after the pandemic.

The pandemic has also exposed the precarious nature of community and youth sport in Australia. Until now, it was not uncommon for talented youth athletes and young adults to 'play for money' in country and amateur sporting competitions (Agnew et al., 2019). A "spotty" funding model of sponsorship, junior fundraising, and government grants to provide 'sporting programs' that attracted talented athletes to the club underpinned the participatory model. However, it is anticipated that many clubs will struggle to generate revenue from sponsors who are likely to endure a recovery period after the pandemic recedes. Clubs may be unable to attract young athletes seeking remuneration for their talent if they have been forced to 'pay back' sponsor money and registration/uniform fees to families and players. Furthermore, player payments may cease to exist altogether if clubs were already in a deficit or had outstanding accounts (e.g. overdue invoices from training and equipment suppliers). Idealistically, it presents an opportunity to rearticulate a culture about why individuals participate in youth sport; but pragmatically, it is likely that many athletes will leave sport altogether if they are not 'paid' for their time and efforts. It remains a harsh, but largely unspoken truth about community sport.

Taken together, these intrapersonal, interpersonal and environmental challenges present immediate risks to youth sport once the threat of COVID-19 recedes. If the infrastructure, resources and programs no longer exist, many who intended to rejoin sport may soon be lost as well. We do know that historically, sporting clubs and organizations are resilient and that many have emerged triumphant through world wars, economic depression and recessions. The difference now is that sporting clubs play a multifaceted role in the community for its participants and it is stakeholders.

Beyond this context, we already know that sport dropout rates for adolescents are high and higher among girls (Drummond et al., 2020). Participation in sport plays a key role in a healthy lifestyle and youth sport is usually a recognised government priority, so any mandated cancellation of sport is particularly concerning. Furthermore, accruing physical activity through sport is an important outcome of participation, but equally important are the social and psychological outcomes associated with sustained engagement with sport. For children (5-18 years), these include fewer depressive symptoms, lower suicide ideation and improved mental health and wellbeing (see Eime et al., 2013). Ongoing engagement in sport and physical activity provides children with life-long development of crucial skills and capabilities, which in turn provides an enormous social, cultural and economic benefit to families, communities and the nation.

We hypothesize that the longer sporting clubs and organizations are not operational as a result of the COVID-19 pandemic, the greater the likelihood that young people will be 'lost' to the sport they once played, and potentially sport in general. The nation will not only lose a critical mass of participants and talented athletes, but also a plethora of young people who would otherwise be engaged in the physical aspects of sport in broader society and culture. Young people's mental wellbeing is also at stake given the fundamental role of sporting clubs, and the support networks that exist within them, in promoting social and emotional support. Therefore the basis of this research will seek to explore the core question that asks "what do you think is needed to reengage young people after COVID-19 is gone"?

The time to investigate this exceptional circumstance is now in order for the sport industry, and those who are socially, culturally and economically invested in its survival, to prepare for 'life' after the pandemic. As a consequence we have developed a 
team to explore the critical issues that will assist sporting clubs, organizations, key stakeholders as well as parents to understand the key issues at hand as well as provide the basis upon which they can mitigate the losses of youth participants in theirs sports, re-engage these participants in the sport and potentially find ways to access new members.

\section{Protocol}

\section{How the sample is to be selected}

This research will be conducted in South Australia given the proximity to the researchers and the relationships we have built with key sporting bodies and organizations. However, South Australia, as the name would suggest, sits at the bottom and towards the middle of mainland Australia. It is uniquely positioned in terms of it being a central post that separates the eastern states of Victoria, New South Wales and Queensland with that of Western Australia, which has the largest landmass of all states and territories in Australia. All of these states, including South Australia are the primary national sporting pathway providers in terms of participants and the need to strengthen the relationships between these pathway providers is paramount (Commonwealth of Australia, 2010). South Australia, while somewhat smaller than that of the eastern states is well placed to create a model for understanding the issues facing sporting clubs given that it maintains the same systems and processes where sporting clubs and organizations are concerned. Like all areas around Australia, the role of the community and volunteerism plays a significant part of the sporting process, and this is integral to community sport in South Australia. Importantly the smaller scale, South Australian based, research allows the research team to easily access participants through sporting clubs and organizations with which we have developed lasting relationships through previous sport based research. As a team we have built a strong record in developing industry-based research with significant research outcomes (Drummond et al., 2019; Drummond et al., 2020).

We intend to focus on young people between the ages of 15-18 as this is the key age group who are at most risk of abstaining or dropping out of sport completely in the wake of a significant hiatus in sporting training or competition. It is at this stage of their lives that young people develop a sense of autonomy and seek to explore alternative interests and opportunities without the same guidance from their parents as when they were younger. Inclusion criteria will consist of any young person between the ages of 15-18 who was involved in sport at the time of the beginning of the COVID-19 pandemic from the period of March $1^{\text {st }}$ 2020. Exclusion criteria will be based on 15-18 year old youth who have not been involved in formal sport for since the beginning of 2020 .

\section{Interventions to be measured}

\section{Phase 1}

We propose to undertake two significant; South Australian based state wide quantitative surveys (using the online platform Qualtrics) to ascertain the impacts of COVID-19 on a range of sports in South Australia. The first survey will target parents of sporting participants as well as all CEOs and Presidents (sporting leaders) of sporting organisations in South Australia, while the second will seek data from young people (15-18 years) to explore the impact at the participant level where drop out is high (Drummond et al., 2020). The two surveys will provide cross sectional data from perspective in terms of those who "run" the sports and those who participate in the sports to measure the impact of COVID-19 on South Australian sport. The survey will be promoted through local sporting networks across South Australia as well as through social media platforms. The surveys will collect descriptive data about age, gender, socio-demographic details such as postcode, sport/ $\mathrm{s}$ in which they participate, commitment to the sport (e.g. local, state, national level), reasons for participating in sport (e.g. goals, fitness, social interaction) reasons for dropping out, and intentions to return to sport following COVID-19. It will also include measures of resilience (Smith et al., 2008) and athletic identity (Altobello, 2006). For sporting clubs the surveys will seek to gather data on club participation levels, clubs structures and governance and explore strategies to mitigate dropout and to increase sport participation post COVID-19 etc. These questions are currently being developed and will be piloted with a group of 15-18 year old participants, together with key stakeholders.

\section{Phase 2}

We intend to undertake qualitative interviews and focus groups with key stakeholders of sporting club and organisations, sporting participants and those who might likely abstain from the sport upon the completion of the COVID-19 pandemic. In this instance we will establish focus groups and interviews through online means such as Zoom, Adobe Connect, Skype or Microsoft Teams app. Given the COVID-19 circumstances we would argue this method will be embraced by the research cohort. In this section we will hold 5 focus groups each with 5 participants $(n=25)$ and 10 individual interviews $(n=10)$. Participants in Phase 1 who have checked the box and consented to be contacted for Phase 2 interviews and/or focus groups will be contacted to establish a time and venue for the interview/focus group. An interview guide will be used. However, this will be devised upon completion of Phase 1, which will inform the development of the Interview and focus group guides.

\section{Sample size calculation}

According to $G^{*}$ Power (Faul et al., 2007), to detect a small effect with power of 0.80 , and an alpha of 0.05 , across the four potential age groups $(15,16,17, \& 18$ years $)$ in the youth sample, a sample size of 1096 is required. For the key stakeholders, to detect a small to medium effect with power of 0.80 , and an alpha of 0.05 across gender a sample size of 156 is required. As such, we will aim to recruit at least 1100 respondents from youth participants and 200 key stakeholders $(\mathrm{N}=1300)$. This sample size should allow us to obtain a representative sample (or cross-section) of youth sport participants and key stakeholders across South Australia including metropolitan and rural and regional South Australia.

\section{Data analysis and statistical plan}

Data will be downloaded from Qualtrics directly to the Statistical Package for the Social Sciences (SPSS) software 25.0 (SPSS, 2017) to analyse the quantitative phase. These data will be screened for normality and no imputations will be used for missing data (Enders, 2010; Tabachnick \& Fidell, 2007). 
Descriptive statistics will be used to describe the sample (i.e., means and percentages), and inferential statistics will be used to establish any significant differences between groups (e.g. by gender or age group). Specifically, for both the youth sample and the key stakeholders, t-tests will be used to examine any differences across gender for any of the continuous outcome measures, and chi-square analyses will be used for categorical outcome measures. For the youth sample age will be split into four groups and we will use ANOVA to examine differences across the different ages involved in the study $(15,16,17,18$ years). Regression will be used to examine any predictors of intentions to return to sport.

Regarding the qualitative data once the focus groups and interviews are transcribed and open coded (Strauss \& Corbin, 1998) they will be inductively analysed to identify major themes. Patton (2002) claims inductive analysis to be a useful mechanism within open-ended discussions, as the inquirer is provided the opportunity to understand patterns within the phenomenon under investigation. Further, Patton notes that categories, patterns and themes can emerge through the interaction with one's data through this form of analysis. Therefore with respect to the data within this research inductive analysis will be based on the teams' previous research undertaken in the area of sport, our professional knowledge and the literature (Strauss, 1987).

\section{Ethical considerations}

The team is based within the Sport, Health, Activity, Performance and Exercise Research Centre where the team members have a strong history and demonstrable track record working with key sporting organisations including the South Australia, National Football League, South Australian Cricket Association, Swimming SA to name a few as well as key government bodies including the Office for Recreation, Sport and Racing (SA), SA Health and the Department for Education. These existing relationships will ensure that accessing research cohorts will not be an onerous task and will provide the opportunity to expedite the process where research data can be collected and research findings disseminated. The team has a recent strong record in conducting large mixed methods research and has research assistants and academics with research expertise and capacity that can be directly transferrable to this priority project. In order to accelerate the process we have already submitted and received full ethics approval from the Flinders Social and Behavioural Ethics Committee (8647 HREC approval notice). All of the survey participants in Phase 1 will be asked to check a box to provide consent to use their anonymous data. All of the Phase 2 participants will be provided with a consent form to sign prior to interviews/focus groups if conducted face-to-face, or verbal consent if conducted online. Audio-recorded data will be transcribed verbatim and then the data will be stored in a secure cloud facility for a period of 5 years and then deleted.

\section{Dissemination of information}

Upon completion of the study the research findings will be disseminated in both traditional and non-traditional formats. Journal articles in SJR Q1 journals will be the core of the dissemination process directed towards the academic community. However, given that the research is highly practical in it application the research will be disseminated in a visual report style to key governments porting organisations as well as offered online to all the sporting community in South Australia and beyond. It is important that we as academics share our research in the interest of collegiality so that we may work collectively and build on ideas, research and interventions globally. This a time when we must trust each other to work together in the academic community to limit the "fallout" that may occur to youth regarding sport and physical activity.

\section{Study status}

The study is in Phase 1. It has recently been provided ethics approval and therefore we are in the process of appointing a part-time research assistant to assist in the development and construction of the online Qualtrics survey. We will soon be sending out a call for participants to be involved in the Phase 1 survey.

\section{Conclusions}

The outcome from this research will provide invaluable data to "arm" government organisations with the facts to establish funding and guidelines to assist key sporting organisations as well as "grassroots" clubs with the necessary resources to establish their sporting environment once again. The need to limit the amount of young people moving away from sports, and the clubs that they represent, needs to be maintained for them to seamlessly transition back into the sport. Otherwise there will be a generation lost to sport, which has major implications for physical, social and mental health as well as creating a cultural void for the nation in terms of the position in which these clubs sit within their local communities. The situation could be potentially far worse in rural Australia where many years of drought and the recent catastrophic bushfires, now coupled with this COVID-19 pandemic could mean that some sporting clubs may cease to exist in the country. Sporting clubs are at the heart of rural Australia. However, this COVID-19 pandemic could be the tipping point for many sporting clubs. This research will provide invaluable insight at a time when a response is immediately required.

\section{Data availability}

Underlying data

No data are associated with this article
Agnew D, Henderson P, Marks A, et al: Maintaining Professionalism in SemiProfessional Sport: Are We Asking too Much? J Glob Sport Manag. 2019; 4(1): 8-24

Publisher Full Text
Altobello S: Toward an Expanded Measure of Athletic Identity: The Inclusion of Public and Private Dimensions. J Sport Exerc Psychol. 2006; 28(4): 434-453.

Publisher Full Text 
Australian Bureau of Statistics (ABS). 2020. Reference Source

Australian Bureau of Statistics (ABS). 2012.

Reference Source

Australian Football League. 2020.

Reference Source

Caple H, Greenwood K, Lumby C: What league? the representation of female athletes in Australian television sports coverage. Media International Australia. 2011; 140: 137-146.

Publisher Full Text

Commonwealth of Australia: Australian Sport: The pathway to success. 2010.

Reference Source

Cuskelly G: Volunteer retention in community sport organisations. European Sport Management Quarterly, 2004; 4(2): 59-76.

Publisher Full Text

Dowell TL, Waters AM, Usher W, et al.: Tackling Mental Health in Youth Sporting Programs: A Pilot Study of a Holistic Program. Child Psychiatry Hum Dev. 2020. PubMed Abstract | Publisher Full Text

Drummond M, Elliott S, Drummond C, et al.: Inclusive Sport Practices: "You don't include people by excluding other people". Flinders University. 2019

Publisher Full Text

Drummond M, Elliott S, Drummond C, et al:: Young people in Sport. Final report. A report for the Office for Recreation, Sport and Racing, SA. 2020

Drummond $\mathrm{M}$, Drummond $\mathrm{C}$, Elliott $\mathrm{S}$, et al.: Promoting healthy physical activity and nutrition in low SES communities: Reflections on a University-Australian football collaborative model. In: Sports-Based Health Interventions: Case Studies from Around the World. Springer. 2016; 279-290.

Reference Source

Eime RM, Young JA, Harvey JT, et al: A systematic review of the psychological and social benefits of participation in sport for children and adolescents: informing development of a conceptual model of health through sport. Int Behav Nutr Phys Act. 2013; 10: 98.

PubMed Abstract | Publisher Full Text | Free Full Text

Elliott S, Bevan N, Litchfield C: Parents, girls' and Australian football: a constructivist grounded theory for attracting and retaining participation.

Qualitative Research in Sport, Exercise and Health. 2019; 1-22.

Publisher Full Text

Enders CK: Applied missing data analysis. Guilford Press. 2010.

Publisher Full Text
Faul F, Erdfelder E, Lang AG, et al:: G*Power 3: A flexible statistical power analysis program for the social, behavioral, and biomedical sciences. Behav Res Methods. 2007; 39(2): 175-191.

PubMed Abstract | Publisher Full Text

Ferrer J, Turner P: Indigenous player inclusion in the Australian Football League. Equality, Diversity and Inclusion: An International Journal. 2017; 36(6): 519-532.

Publisher Full Text

Haycraft JAZ, Kovalchik S, Pyne DB, et al:: Physical characteristics of players within the Australian Football League participation pathways: a systematic review. Sports Med Open. 2017; 3(1): 46.

PubMed Abstract | Publisher Full Text | Free Full Text

Holt NL, Kingsley BC, Tink LN, et al.: Benefits and challenges associated with sport participation by children and parents from low-income families. Psychology of sport and exercise. 2011; 12(5): 490-499.

Publisher Full Text

IBM Corp: IBM SPSS Statistics for Windows. Armonk, NY: IBM Corp. 2017.

Jeanes R, Spaaij R, Magee J, et al.: Developing participation opportunities for young people with disabilities? Policy enactment and social inclusion in Australian junior sport. Sport in Society. 2019; 22(6): 986-1004.

Publisher Full Text

Jeanes R, Spaaij R, Penney D, et al.: Managing informal sport participation: tensions and opportunities. International Journal of Sport Policy and Politics.

2019; 11(1): 79-95.

Publisher Full Text

Patton M: Qualitative research and evaluation methods. ( $3^{\text {rd }}$ Ed). Thousand Oaks California: Sage. 2002

Reference Source

Smith BW, Dalen J, Wiggins K, et al:: The Brief Resilience Scale: Assessing the Ability to Bounce Back. Int J Behav Med. 2008; 15(3): 194-200.

PubMed Abstract | Publisher Full Text

Strauss AL: Qualitative analysis for social scientists. Cambridge: Cambridge University Press. 1987

Publisher Full Text

Strauss A, Corbin J: Basics of qualitative research. Second edition. Newbury Park, California: Sage. 1998.

Reference Source

Tabachnick BG, Fidell LS: Using Multivariate Statistics. 5th edn. (Allyn and

Bacon: Boston, MA.). 2007.

Reference Source 


\section{Open Peer Review}

\section{Current Peer Review Status: $\mathrm{X} \times$}

Version 1

Reviewer Report 22 March 2022

https://doi.org/10.21956/emeraldopenres.14735.r27970

(C) 2022 Wold B. This is an open access peer review report distributed under the terms of the Creative Commons Attribution License, which permits unrestricted use, distribution, and reproduction in any medium, provided the original work is properly cited.

\section{Bente Wold}

Department of Health Promotion and Development, Faculty of Psychology, University of Bergen, Bergen, Norway

This study protocol addresses an interesting and important issue; the consequences of the Covid19 pandemic on participation in sport among young people. The authors maintain that many sports clubs are not well prepared for the effects of a pandemic that has led to a temporary interruption of activities, and that many young athletes may be lost to sport. They argue that it is necessary to develop strategies to re-engage parent volunteers in youth sport and propose several challenges that need to be tackled. The main research question is 'what do you think is needed to reengage young people after COVID-19 is gone?'. The protocol describes plans for two South Australian statewide quantitative surveys, and qualitative interviews and focus groups with key stakeholders and sporting participants likely to abstain from participation after COVID-19.

The authors should be commended for seeing the potential in studying COVID-19 related opinions and feelings of key contributors to youth sport at the time of the pandemic. Planning to use different methods and various types of informants is also a strength of the study.

While several of the statements concerning the potential effects of the pandemic seem sensible, they are not clearly substantiated with theoretical or empirical support. This part of the paper therefore seems speculative. There is a confusing mix of arguments linked to either recruitment to elite sport or to inclusive health promoting youth sport. My advice is to select one of these, as there may be very different mechanisms underlying recruitment and continuation of young people in the first versus the latter. These differences are likely to result in different consequences of the pandemic, which in turn will result in the need for different types of interventions/actions.

Most of the arguments and background of the study seem to be directed at what sport clubs and organizations can do to mitigate the potential negative effects of activities being cancelled due to COVID-19 measures. It would be good to follow up this question in the methods, by focusing more closely on key stakeholders in clubs. The planned surveys among parents and young people seem to give other types of information that is not sufficiently related to this question. Thus, the methods are only partially adequate, and the study protocol seems overall vague and unclear. 
The planned surveys may give different types of information depending on the timing of the surveys with regard to the status of the pandemic measures. If the surveys are undertaken when society has reopened, or at least when activities in youth sport clubs are available again, parents, key stakeholders and youth may respond in a very different way than if the clubs and activities are closed. For example, if the clubs are operational, questions about intentions to return to sport may be less relevant as participants may already have returned. It is therefore vital that the impact of timing is taken into consideration.

In the focus groups and interviews, 'those who might likely abstain from sport' are to be included, but it is not clear how these individuals will be identified. It doesn't seem possible to find those who are not active and 'blame' the pandemic for that, as they may not have been participating in sport regardless of COVID-19 consequences.

The study may give interesting information about opinions about how COVID-19 measures may have impacted participation in youth sport. However, it will not be possible to infer whether reengagement of young people will be a consequence of efforts to mitigate the effects of the pandemic or just the common reasons for drop-out. If drop-out levels are higher after the pandemic than before, perhaps there are data available that can be used as comparative, standardized or normative data?

Is the rationale for, and objectives of, the study clearly described?

Partly

Is the study design appropriate for the research question?

Partly

Are sufficient details of the methods provided to allow replication by others?

No

Are the datasets clearly presented in a useable and accessible format?

No

Is the argument information presented in such a way that it can be understood by a nonacademic audience?

Yes

Does the piece present solutions to actual real world challenges?

Yes

Is real-world evidence provided to support any conclusions made?

No

Could any solutions being offered be effectively implemented in practice?

Not applicable

Competing Interests: No competing interests were disclosed. 
Reviewer Expertise: Youth sport, health promotion, developmental psychology

\section{I confirm that I have read this submission and believe that I have an appropriate level of expertise to state that I do not consider it to be of an acceptable scientific standard, for reasons outlined above.}

Reviewer Report 01 June 2021

https://doi.org/10.21956/emeraldopenres.14735.r27520

(C) 2021 Morgan H. This is an open access peer review report distributed under the terms of the Creative Commons Attribution License, which permits unrestricted use, distribution, and reproduction in any medium, provided the original work is properly cited.

\section{Haydn Morgan}

Department for Health Centre for Qualitative Research, University of Bath, Bath, United Kingdom

This study protocol contains much promise and appears (on the surface at least) to address one of the most substantial challenges related to sport as a result of the COVID-19 pandemic. However, the proposal lacks important detail and requires more justification for its overarching aims, focus and intended contribution to knowledge.

In my view, the title and Abstract are slightly misleading in their intentions. From my reading, the proposal is more focussed upon community sports clubs and how they intend to re-emerge/reengage once the pandemic ceases to be considered a public health problem. Therefore, the focus of the title, Abstract, and significant parts of the Introduction, on a 'lost generation' seems off the mark, and perhaps is designed to sensationalise the research. I have no concern with the research exploring youth drop-out from sport, but for me, the emphasis should be on the perspectives of clubs. I am also quite uncomfortable about the use of the term 'lost generation', particularly in the broader context of Australian history and politics, so I would suggest a moderation of this terminology. The Introduction (and Abstract) are based on an assumption that the pandemic will create a 'lost generation' of young people, which appears to be a rather speculative assumption. The Introduction requires more substantiation from the literature (which in itself is mostly dated) for the assumptions that the research seeks to address. This will lead to more logical and substantive aims (which currently are very vague - the core question "what do you think is needed to reengage young people after COVID-19 is gone"? is vague - who is 'you', for example).

The literature review is broad reaching, focussing on several connected issues apparent in the sporting context. However, the proposal needs to be clear on which ones the current study will focus upon. The literature review describes several challenges for the sport community that result from the pandemic. While most are valid claims, they would be better substantiated if more literature was incorporated - at present many of these claims are presented as 'personal opinion' or 'received wisdom'. Also, I wonder how many of the challenges have always been present (or were present) prior to the pandemic? For example, the challenge of recruiting volunteers has long been a challenge, so I am not sure how 'the necessity to return to work once the pandemic recedes' is any different to before. Also, high cost sporting activities have long been 'out of the reach' of many families/individuals (see Thibault et al., $1993^{1} ; 1994^{2}$ for an early exploration of this 
topic), so again, an explanation of how the pandemic exacerbates this issue specifically is needed. In my view, this will enable the derivation of a specific research question(s) - as noted the current question, which is hidden away, is extremely vague.

In terms of the methodological aspects, there are several areas that require tightening. In the section on sampling (p.5), I am not convinced how the geography of the selected state makes it uniquely positioned as a research site? I would suggest related sentences are removed. It appears that the authors are in some way concerned that their study relies on convenience sampling (based upon re-engaging previous participants). I see no problem with convenience sampling, provided the justification for its use is argued. Further comments on the sample -1 ) a reference is needed to support the claim about peak drop being between the ages 15-18; 2) more detail on the inclusion (exclusion) criteria is needed - e.g. what does being 'involved in (formal) sport' mean? And why is formal sport mentioned in the exclusion criteria but not the inclusion criteria? 3) The section on sample size calculation would appear to be more salient in the section on sampling; 4) The opening sentences in the 'Ethical considerations' section (p. 6) regarding existing connections to sporting organisations would be better placed in the section on sampling. Further detail here would help with providing justification for the feasibility of meeting the sample sizes noted in point 3) above.

Phase 1: It is suggested that two surveys will be used, with the first targeted at 'parents of sporting participants, as well as sporting leaders'. Why are these participants not mentioned in the section on sampling? Phase 1 also noted that the 'youth focussed survey' will contain items on 'resilience' and 'athletic identity'. Neither of these terms is noted in the literature review, so some reflection on why these concepts are relevant to the research is needed at some point (I would suggest in the literature review).

Phase 2: It is suggested that interview participants will be selected from 'those who might likely abstain from the sport [sic]'. How will this sample be determined/established? What would constitute a participant to be a 'likely abstainer'?

Dissemination/Conclusion: The proposal needs to be more precise in its intended reach - 'South Australia and beyond' seems a little prosaic. Some attempt is made in the Conclusion to offer more detail - 'providing invaluable data to 'arm' government organisations with facts' - but without opening an ontological and epistemological debate on evidence and knowledge, I would encourage the authors to moderate their language in this statement, particularly the use of the word 'facts'. The Conclusion also draws attention to 'major implications for physical, social and mental health'. I would wholeheartedly agree with this, but the case for these implications has not been established with any detail, and should be a central element of the literature review. The same goes for the comment about 'rural Australia' which is only introduced in the Conclusion, but should play a much stronger part in the initial rationalising of the project to demonstrate the social and political importance of this research, and thus position it as timely and meaningful.

Finally, the proposal would benefit from a thorough proof-read, both for accuracy and the omission of any non-academic/colloquial language.

\section{References}

1. Thibault L, Slack T, Hinings B: A Framework for the Analysis of Strategy in Nonprofit Sport Organizations. Journal of Sport Management. 1993; 7 (1): 25-43 Publisher Full Text 
2. Slack T, Hinings B: Strategic Planning for Nonprofit Sport Organizations: Empirical Verification of a Framework. Journal of Sport Management. 1994; 8 (3): 218-233 Publisher Full Text

Is the rationale for, and objectives of, the study clearly described?

Partly

Is the study design appropriate for the research question?

Partly

Are sufficient details of the methods provided to allow replication by others? No

Are the datasets clearly presented in a useable and accessible format?

No

Is the argument information presented in such a way that it can be understood by a nonacademic audience?

Not applicable

Does the piece present solutions to actual real world challenges?

Yes

Is real-world evidence provided to support any conclusions made?

Not applicable

Could any solutions being offered be effectively implemented in practice?

Not applicable

Competing Interests: No competing interests were disclosed.

Reviewer Expertise: Sport, volunteering, community development, youth exclusion.

I confirm that I have read this submission and believe that I have an appropriate level of expertise to state that I do not consider it to be of an acceptable scientific standard, for reasons outlined above. 